\title{
Malfunctions in synaptic membrane trafficking in early pathology of Parkinson's disease: New molecular clues
}

\author{
Elena Sopova ${ }^{1,2}$, Olga Korenkova², and Oleg Shupliakov ${ }^{1,2}$ \\ 1Department of Neuroscience, Karolinska Institutet, 17177 Stockholm, Sweden; \\ ${ }^{2}$ Institute of Translational Biomedicine, Saint Petersburg State University, \\ Universitetskaya nab., 7-9, Saint Petersburg, 199034, Russian Federation \\ Address correspondence and requests for materials to Oleg Shupliakov, \\ oshupliakov@yandex.ru
}

\section{Abstract}

The midbrain dopaminergic neurons of the substantia nigra and the ventral tegmental area play vital roles in the regulation of voluntary movement, emotion and reward in humans. These neurons are highly metabolic and are under constant oxidative stress. The dopaminergic neurons form extensive synaptic projections to the striatum. When these neurons start dying or when their synaptic connections fail, humans develop Parkinson's disease. This disease is accompanied by the accumulation of toxic a-synuclein-containing protein aggregates in nigrostriatal processes. Synucleins accumulate in a majority of healthy nerve terminals in the central nervous system, but what causes the formation of pathological synuclein aggregates is unclear. Recent studies point out that the interface between membrane trafficking in the nerve terminal and the autophagy-lysosomal pathway is the site for the aggregate assembly. An urgent goal is to find therapeutic targets at early stages of the disease when neurons are still functional.

Keywords: synapse, synaptic proteins, atophagy-lysosomal pathway, developmental transcription factors, Parkinson's disease.

Citation: Sopova, E., Korenkova, O., and Shupliakov, O. 2017. Malfunctions in synaptic membrane trafficking in early pathology of Parkinson's disease: New molecular clues. Bio. Comm. 62(4): 272-277. https://doi.org/10.21638/11701/ spbu03.2017.406

Author's information: Elena Sopova; Olga Korenkova; Oleg Shupliakov, Ph.D. orcid.org/0000-0001-5352-6848

Manuscript Editor: Mikhail Kostylev, Department of Neurology, Yale University, USA;

Received: January 28, 2018;

Revised: April 19, 2018 ;

Accepted: April 20, 2018;

Copyright: ( 2017 Sopova et al. This is an open-access article distributed under the terms of the License Agreement with Saint Petersburg State University, which permits to the authors an unrestricted distribution and self-archiving free of charge.

Funding: This work was supported by the Russian Science Foundation (Grant No. 16-15-10273), the Swedish Medical Research Council (Grant No. VR-M №1501), and Parkinsonfonden.

Competing interests: The author has declared that no competing interests exist.
Parkinson's disease (PD) is a progressive neurodegenerative disease characterized by the loss of nigrostriatal neurons, which results in a triad of classical symptoms, such as resting tremor, rigidity and hypokinesia; these symptoms are followed by alterations in gait and balance and additional disturbances (Lotharius and Brundin, 2002; Cookson and Bandmann, 2010; Schulz-Schaeffer, 2012; Olanow and Brundin, 2013; Papandreou and Tavernarakis, 2017). Parkinson's disease is one of the most common neurodegenerative diseases. According to official statistics the estimate of the number of patients with PD in 2016 in Russia was approximately 210.000 people (Razdorskaya, Voskresenskaya, and Yudina, 2016). The disease resulted in about 103,000 deaths worldwide in 2013 (Parkinson's disease, 2018). Currently there is no medication that can effectively stop the disease onset and progression.

Among the first neurodegenerative changes that occur in PD in the brain is a loss of dopaminergic nerve terminals in the striatum, accompanied by the accumulation of $\alpha$-synuclein-containing protein aggregates in nigrostriatal processes known as Lewy neuritis (Lotharius and Brundin, 2002; Braak et al., 2003; Hansen and $\mathrm{Li}, 2012$ ). The appearance of the inclusions in nerve terminals in $\mathrm{PD}$ is followed by retrograde degeneration, further accumulation of Lewy bodies in cells in substantia nigra (SN), and finally cell death (Lotharius and Brundin, 2002; Cookson and Bandmann, 2010; Schulz-Schaeffer, 2012; Olanow and Brundin, 2013). 
It has been suggested that the death of neurons in $\mathrm{SN}$ involves dopamine-dependent oxidative stress; over the last decade much of the research on PD has focused on cellular stress, which has been proposed as a candidate mechanism leading to degeneration of neurons (Lotharius and Brundin, 2002; Cookson and Bandmann, 2010; Guzman et al., 2010; Schulz-Schaeffer, 2012; Olanow and Brundin, 2013). Recent studies, however, have provided evidence that other molecular and cellular mechanisms may be involved. Identification of PD-related mutations in the PARK2 gene, which encodes the E3 ubiquitin ligase, parkin, and PARK5, which encodes the deubiquitination enzyme Ubiquitin carboxy-terminal hydrolase L1, shifted attention from the pathological consequences of misfolded synuclein to the malfuctions in the ubiquitin proteosome system and autophagy-lysosome pathway. It has been reported, for example, that parkin might affect $\alpha$-synuclein function by participating in the ubiquitination of the a-synuclein interacting proteins: synphilin 1 (Chung et al., 2001; Engelender, 2008), CDCrel 1 also referred to as septin 5 (Zhang et al., 2000), and septin 4 (Shehadeh et al., 2009), which might cause reduced degradation of these proteins. Supporting this, numerous additional proteins - e.g., ubiquitin, proteosome subunits, heat-shock proteins, and neurofilaments - were reported in Lewy bodies (Lotharius and Brundin, 2002; Luk et al., 2012). Mutations in genes such as DJ1, ATP13A2 and PINK have implicated mitochondrial dysfunctions in disease progression (Cookson and Bandmann, 2010). All these studies shifted the scientific focus from synaptic functions to the cell body and mitochondrial stress pathways as the central aspect of the pathogenesis for some time.

Several recent publications have brought the spotlight back to presynaptic terminals (Esposito, Ana Clara and Verstreken, 2012; Heutink and Verhage, 2012; Matta et al., 2012). It has been proposed that $\alpha$-synuclein aggregates derive from perturbations of the normal functions of synucleins in synaptic membrane trafficking (Burre, Sharma and Sudhof, 2012). Consistent with the membrane-associated function of synucleins in nerve terminals, transgenic mice overexpressing human a-synuclein displayed alterations of the internal synaptic membrane morphology (Boassa et al., 2013). Synapses studied in 3D using electron microscopy were enlarged, contained endosome-like structures and numerous tubulovesicle structures, and in many cases were filled with membrane-bound organelles.

$\alpha$-synuclein belongs to the synuclein protein family $(\alpha, \beta, \gamma)$, which is only expressed in vertebrate species. $\alpha$ and $\beta$-synucleins are highly homologous and enriched in nerve terminals (Maroteaux, Campanelli and Scheller, 1988; Cookson and Bandmann, 2010). Native cell-derived $\alpha$-synuclein is a tetramer in solution, and this form has greater lipid-binding capacity than recombinantly expressed monomers (Bartels, Choi and Selkoe, 2011). Multiplications of the gene locus encoding $\alpha$-synuclein, SINCA (synuclein, alpha nonA4 component of amyloid precursor), or mutations in the gene cause rare familial dominant $\mathrm{PD}$, while single nucleotide polymorphisms in the SINCA gene have been identified to be associated with sporadic PD. a-synuclein forms oligomers referred to as protofibrils that can seed in a nucleation-dependent manner to form the amyloid fibrils. Amyloid fibrils have been found in vivo in a-synuclein-containing protein aggregates (referred to as Lewy bodies) in nigrostriatal processes and neurons in PD (Lotharius and Brundin, 2002; Olanow and Brundin, 2013).

Naturally, $\alpha$-synuclein is one of the prime targets in the search for treatments for PD (Rivero-Rios, MaderoPerez, Fernandez and Hilfiker, 2016; Moors et al., 2017). Peptide-protein conjugate vaccines, designed to elicit neutralizing selective antibodies against $\alpha$-synuclein, are currently in clinical trials for acute treatments of the disease (Mandler et al., 2014). It should be taken into consideration, however, that $\alpha$-synuclein is involved in the modulation of synaptic transmission (Scott et al., 2010; Scott and Roy, 2012; Vargas et al.m 2014). It is therefore important to know the exact physiological functions of this protein in healthy nerve terminals and how it may contribute to the onset and progression of PD pathology before such antibodies are broadly used in clinical practice.

Synucleins are accumulated in contacts established by synapses of different modalities in the central nervous system (CNS). A number of roles for $\alpha$-synuclein in the synaptic vesicle (SV) cycle have been proposed. It has been shown that $\alpha$-synuclein restricts the lateral mobility of synaptic vesicles between synaptic boutons along the axon (Staras et al., 2010; Scott and Roy, 2012). Small increments in a-synyclein levels lead to suppression of the exo-endocytic cycle (Scott et al., 2010; Scott and Roy, 2012). Endocytosis is inhibited in synuclein $\alpha$, $\beta, \gamma$-triple KO mice, and physiological kinetic studies allowed suggesting that synuclein contributes to the progression of early stages of SV endocytosis (Vargas et al., 2014). Lipid-binding properties of $\alpha$-synuclein and its ability to interact with the endocytic adaptor AP180 are consistent with such function.

Protein-protein interaction studies have also predicted a role for synuclein in SV clustering after endocytosis (Wang et al., 2014). Synucleins localize to the SV pool (Wang et al., 2014) and colocalize with SV-associated phosphoproteins, synapsins (Woods et al., 2007). Synapsin I has a well-established function in organizing SV in clusters. $\alpha$-synuclein and synapsin I both contain an amphiphatic lipid packing sensor-motif (ALPS-motif), which binds to curved membranes (Krabben et al., 2011). It cannot be excluded that both proteins contribute to proper SV organization at the synaptic active zone. 
It has been demonstrated that $\alpha$-synuclein multimers cluster SV and restrict their motility in vitro, consistent with localization of the protein in synapses (Maroteaux et al., 1988; Cookson and Bandmann, 2010). How these synaptic functions are linked to the $\alpha$-synuclein amyloid formation is unclear; however, recent data clearly show that formation of $\alpha$-synuclein aggregates in nerve terminals may take place.

Synuclein aggregates were observed in mice with a mutation in the essential regulator of the fusion machinery Munc18-1 linked to epileptic encephalopathy and PD in humans. Munc18-1 binds to the SNARE receptor syntaxin-1A, which serves as a molecular chaperone for $a$-synuclein. The mutant protein coaggregates with a-synuclein (Chai et al., 2016), further suggesting that malfunctions in the SV cycle proteins may initiate $\mathrm{PD}$ related pathology (Fig. 1).

Recently, mutations in two SV uncoating factors, auxilin and synaptojanin-1, were found to cause earlyonset PD. Auxilin is recruited to the clathrin coats due to the action of the endocytic polyinositolphosphatase, synaptojanin1, which is brought to clathrin-coated pits by the key endocytic adaptor endophilin. Recent genetic rescue experiments in mice with a PD-related mutation R258Q in synaptojanin1 did not report any accumulation of protein aggregates at synapses but observed abnormal accumulation of membrane vesicles and folds in $\mathrm{mDA}$, suggesting that other defects in membrane trafficking may also underlay early PD pathology (Boassa et al., 2013; Cao et al., 2017).

The family of proteins, endophilin A1, A2 and A3 (also referred to as endophilin 1,2 and 3) is a subset of the protein superfamily containing Bin/Amphiphysin/ RVS (BAR) domains, which are known to be responsible for sensing and generating membrane curvature, and for recruiting the relevant endocytic factors from the cytosol to the membrane (Saheki and De Camilli, 2012). Endophilin recruits the phosphatase synaptojanin 1 to the necks of the budding vesicle prior to fission by the GTPase dynamin, which also interacts with endophilin, but can act independently of it (Gad et al., 2000; Milosevic et al., 2011). Endophilin 1 also interacts with the synaptic scaffolding protein Intersectin 1, which coordinates the synaptic vesicle cycle and membrane trafficking events outside synapses (Pechstein et al., 2015).

Endophilin is linked to PD and neurodegeneration. It is altered in the cortex of PD patients, and it interacts with two hallmark PD proteins, the E3 ubiquitin ligase parkin and the leucine-rich repeat kinase LRRK2, the most commonly disrupted gene in familial PD (Murdoch et al., 2016; Soukup et al., 2016; Soukup and Verstreken, 2017). Unbiased proteomic screening of brain proteins in mice lacking all three synuclein genes revealed a prominent increase in endophilin 1 levels (Burre et al., 2013). Mouse endophilin triple knockout
(TKO) has a distinct morphological and cellular phenotype characterized by impaired SV recycling, diminished autophagy/altered protein homeostasis, increased apoptosis and gliosis, neurodegeneration, motor impairments and reduced lifespan. A partial loss of endophilin in mice also results in neurodegeneration, ataxia and early lethality (Milosevic et al., 2011; Cao, Milosevic, Giovedi and De Camilli, 2014; Murdoch et al., 2016).

Several recent studies linked endophilin and its endocytic binding partner synaptojanin to the maturation of autophagosomes in the synapse (Murdoch et al., 2016; Soukup et al., 2016; Soukup and Verstreken, 2017), which expanded its role far beyond the SV recycling and synaptic compartment, and allowed linking it to pathological neurodegenerative conditions, including $\mathrm{PD}$, in accordance with the complex phenotype of the endophilin TKO (Fig. 1). Endophilin 1 localizes on autophagosomal membranes and is critical for the maturation of synaptic autophagosomes (Murdoch et al., 2016; Soukup et al., 2016).

The autophagy-lysosomal pathway (ALP) is believed to be the main route for the intracellular degradation of $\alpha$-synuclein. Chaperone-mediated autophagy has been linked to clearance of $\alpha$-synuclein. It has been shown that the protein interacts with the heat shock cognate 70 (Hrs70), which in turn binds to the lysosomal transmembrane protein Lamp2a and facilitates subsequent lysosomal degradation. a-synuclein mutants bind to Lamp2 with higher affinity and block the process of $\alpha$-synuclein degradation (Cuervo et al., 2004). Block of the vacuolar protein-sorting complex Vps34-Beclin1 stops autophagosome formation, which may result in $\alpha$-synuclein accumulation. $\alpha$-synucleincontaining aggregates may also form at later ALP stages (Rivero-Rios et al., 2016; Moors et al., 2017). Mutations in LRRK2, an autophagy regulator kinase interferes with clearance of $\alpha$-synuclein accumulation from autophagosomes. Mutations in PINK1 and parkin perturb proper autophagic clearance of defunct mitochondria, causing a buildup of these organelles and resulting in failure to properly meet metabolic demands. Mutant lysosomal enzyme glucocerebrosidase (GBA) and ATP13A2 decrease lysosomal degradative capacity. In all named mutations, an increase in a-synuclein toxicity is observed, causing $\alpha$-synuclein-mediated autophagic impairment and cellular pathology (Rivero-Rios et al., 2016; Moors et al., 2017).

Control of the ALP pathway is executed by transcription factors. For example, conditional knockout (cKO) of $L m \times 1 b$ has been associated with perturbations in the ALP (Laguna et al., 2015). Lmx1b is involved in postmitotic neuronal regulation of ALP proteins Beclin1, Lamp1-2, p62, cathepsin D and LC3BI-II. It influences expression of transcription factor EB (TEEB), a critical regulator of genes involved in lysosomal bio- 


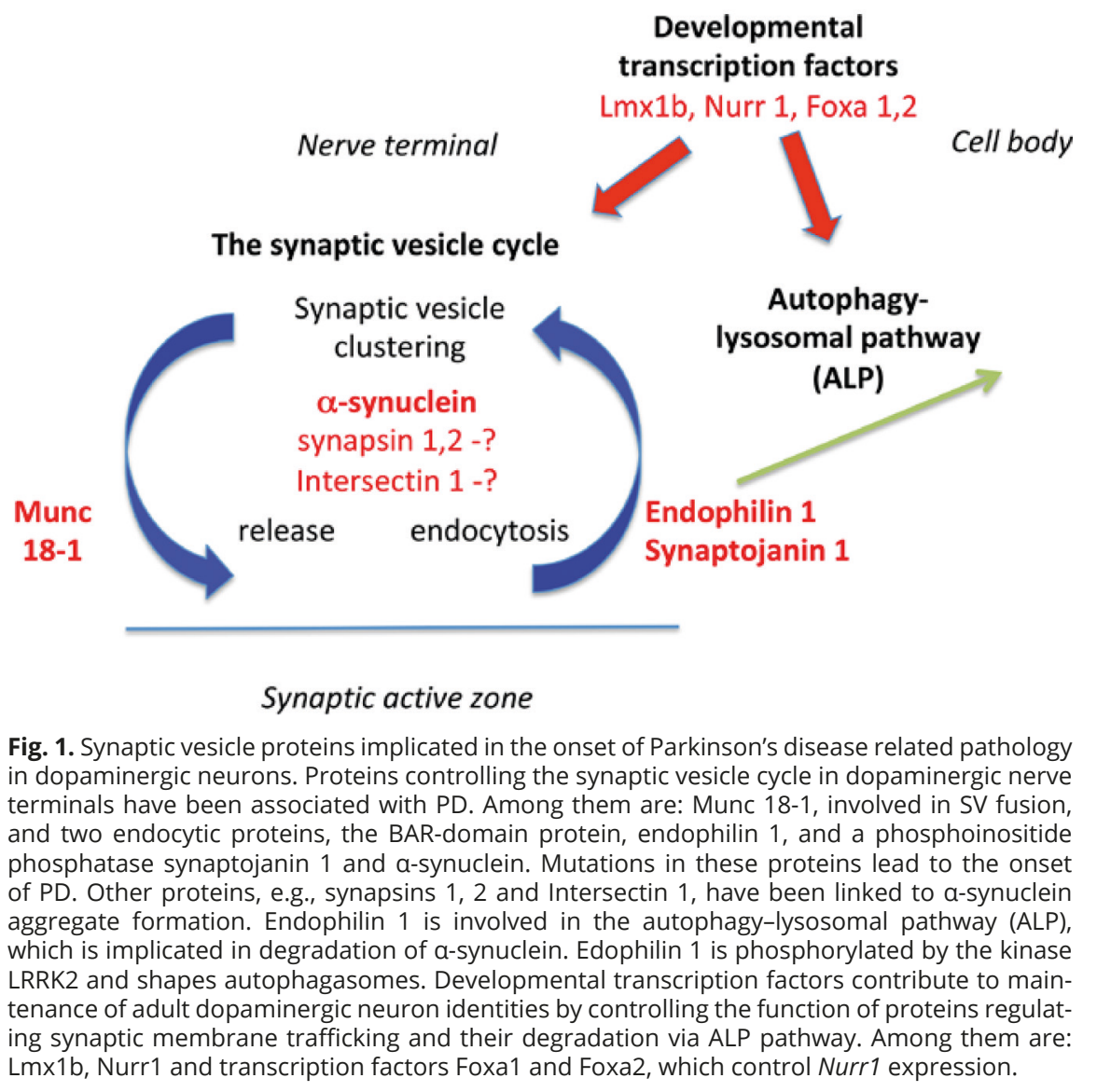

genesis, and Nurr1, which down-regulates expression of proteins involved in dopamine transmission. Conditional targeting of $L m \times 1 b$ in mice leads to dramatic changes in axonal bouton membrane organization, disruption of synaptic morphology, swelling, protein aggregation and reduction in the number of active zones in dopaminergic nerve terminals in striatum. This suggests that dysfunctional interactions between ALP and synaptic membrane trafficking, observed in the animal model, may be involved in PD pathology in humans. Supporting this, postmortem human brain tissue analyses revealed a significant reduction of Lmxlb in samples from PD brains (Laguna et al., 2015). It remains unclear exactly how ALP is related to the synuclein cycle and membrane trafficking events in synapses. Further elucidation of molecular steps leading to the formation of synuclein aggregates in synapses will define the role of autophagy-lysosomal pathway in elimination of the amyloid aggregates.

In conclusion, several recent studies strongly indicate that the elucidation of synaptic membrane trafficking mechanisms linked to PD will lead to identification of novel therapeutic targets at early stages of the disease when many dopaminergic neurons are still functional. In combination with development of new "drug discovery" model systems - for example, differentiated dopaminergic neurons with functional synapses from human induced pluripotent stem (iPS) cells - these studies will lead to selection of medicines for the treatment of Parkinson's disease.

This work was supported by funding from the Russian Science Foundation grant №16-15-10273, the Swedish Medical Research Council (VR-M №1501), and Parkinsonfonden (Sweden).

\section{References}

Bartels, T., Choi, J. G. and Selkoe, D. J. 2011. Alpha-synuclein occurs physiologically as a helically folded tetramer that resists aggregation. Nature 477 (7362):107-110. https://doi.org/10.1038/nature10324

Boassa, D., Berlanga, M.L., Yang, M.A., Terada, M., Hu, J., Bushong, E. A., Hwang, M., Masliah, E., George, J. M. and Ellisman, M. H. 2013. Mapping the subcellular distribution of alpha-synuclein in neurons using genetically encoded probes for correlated light and electron microscopy: Implications for parkinson's disease pathogenesis. Journal of Neuroscience 33 (6):2605-2615. https://doi.org/10.1523/JNEUROSCI.2898-12.2013

Braak, H., Del Tredici, K., Rub, U., de Vos, R. A., Jansen Steur, E. N. and Braak, E. 2003. Staging of brain pathology related to sporadic parkinson's disease. Neurobiology of Aging 24 (2):197-211. https://doi.org/S0197458002000659

Burre, J., Sharma, M. and Sudhof, T. C. 2012. Systematic mutagenesis of alpha-synuclein reveals distinct sequence requirements for physiological and pathological activities. Journal of Neuroscience 32 (43):15227-15242. https://doi.org/10.1523/JNEUROSCI.3545-12.2012 
Burre, J., Vivona, S., Diao, J., Sharma, M., Brunger, A.T. and Sudhof, T.C. 2013. Properties of native brain alphasynuclein. Nature 498 (7453):E4-E6; discussion E6-7. https://doi.org/10.1038/nature12125

Cao, M., Milosevic, I., Giovedi, S. and De Camilli, P. 2014. Upregulation of parkin in endophilin mutant mice. Journal of Neuroscience 34 (49):16544-16549. https://doi.org/10.1523/JNEUROSCI.1710-14.2014

Cao, M., Wu, Y., Ashrafi, G., McCartney, A. J., Wheeler, H., Bushong, E. A., Boassa, D., Ellisman, M. H., Ryan, T. A. and De Camilli, P. 2017. Parkinson sac domain mutation in synaptojanin 1 impairs clathrin uncoating at synapses and triggers dystrophic changes in dopaminergic axons. Neuron 93 (4):882-896 e885. https://doi.org/10.1016/j. neuron.2017.01.019

Chai, Y.J., Sierecki, E., Tomatis, V. M., Gormal, R. S., Giles, N. Morrow, I. C., Xia, D., Gotz, J., Parton, R. G., Collins, B. M., Gambin, Y. and Meunier, F. A. 2016. Munc18-1 is a molecular chaperone for alpha-synuclein, controlling its self-replicating aggregation. Journal of Cell Biology 214 (6):705-718. https://doi.org/10.1083/jcb.201512016

Chung, K. K., Zhang, Y., Lim, K. L., Tanaka, Y., Huang, H., Gao, J., Ross, C. A., Dawson, V. L. and Dawson, T. M. 2001. Parkin ubiquitinates the alpha-synuclein-interacting protein, synphilin-1: Implications for lewy-body formation in parkinson disease. Nature Medicine 7 (10):1144-1150. https://doi.org/10.1038/nm1001-1144

Cookson, M. R. and Bandmann, O.2010. Parkinson's disease: Insights from pathways. Human Molecular Genetics 19 (R1):R21-R27. https://doi.org/10.1093/hmg/ddq167

Cuervo, A. M., Stefanis, L., Fredenburg, R., Lansbury, P.T. and Sulzer, D. 2004. Impaired degradation of mutant alpha-synuclein by chaperone-mediated autophagy. Science 305 (5688):1292-1295. https://doi.org/10.1126/science. 1101738

Engelender, S. 2008. Ubiquitination of alpha-synuclein and autophagy in parkinson's disease. Autophagy 4 (3):372-374

Esposito, G., Ana Clara, F. and Verstreken, P. 2012. Synaptic vesicle trafficking and parkinson's disease. Developmental Neurobiology 72 (1):134-144. https://doi.org/10.1002/ dneu.20916

Gad, H., Ringstad, N., Low, P., Kjaerulff, O., Gustafsson, J., Wenk, M., Di Paolo, G., Nemoto, Y., Crun, J., Ellisman, M. H., De Camilli, P., Shupliakov, O. and Brodin, L. 2000. Fission and uncoating of synaptic clathrin-coated vesicles are perturbed by disruption of interactions with the sh3 domain of endophilin. Neuron 27 (2):301-312.

Guzman, J. N., Sanchez-Padilla, J., Wokosin, D., Kondapalli, J., Ilijic, E., Schumacker, P. T. and Surmeier, D. J. 2010. Oxidant stress evoked by pacemaking in dopaminergic neurons is attenuated by dj-1. Nature 468 (7324):696-700. https://doi.org/10.1038/nature09536

Hansen, C. and Li, J.Y. 2012. Beyond alpha-synuclein transfer: Pathology propagation in parkinson's disease. Trends in Molecular Medicine 18 (5):248-255. https://doi.org/10.1016/j.molmed.2012.03.002

Heutink, P. and Verhage, M. 2012. Neurodegeneration: New road leads back to the synapse. Neuron 75 (6):935-938. https://doi.org/10.1016/j.neuron.2012.09.006

Krabben, L., Fassio, A., Bhatia, V. K., Pechstein, A., Onofri, F., Fadda, M., Messa, M., Rao, Y., Shupliakov, O., Stamou, D., Benfenati, F. and Haucke, V.2011. Synapsin i senses membrane curvature by an amphipathic lipid packing sensor motif. Journal of Neuroscience 31 (49):18149-18154. https://doi.org/10.1523/JNEURO$\mathrm{SCl} .4345-11.2011$

Laguna, A., Schintu, N., Nobre, A., Alvarsson, A., Volakakis, N., Jacobsen, J. K., Gomez-Galan, M., Sopova, E., Joodmardi,
E., Yoshitake, T., Deng, Q., Kehr, J., Ericson, J., Svenningsson, P., Shupliakov, O. and Perlmann, T. 2015. Dopaminergic control of autophagic-lysosomal function implicates $1 \mathrm{~m} \times 1 \mathrm{~b}$ in parkinson's disease. Nature Neuroscience 18 (6):826-835. https://doi.org/10.1038/nn.4004

Lotharius, J. and Brundin, P. 2002. Pathogenesis of parkinson's disease: Dopamine, vesicles and alpha-synuclein. Nature Reviews Neuroscience 3 (12):932-942. https://doi.org/10.1038/nrn983

Luk, K. C., Kehm, V., Carroll, J., Zhang, B., O'Brien, P., Trojanowski, J. Q. and Lee, V. M. 2012. Pathological alpha-synuclein transmission initiates parkinson-like neurodegeneration in nontransgenic mice. Science 338 (6109):949-953. https://doi.org/10.1126/science.1227157

Mandler, M., Valera, E., Rockenstein, E., Weninger, H., Patrick, C., Adame, A., Santic, R., Meindl, S., Vigl, B., Smrzka, O., Schneeberger, A., Mattner, F. and Masliah, E. 2014. Next-generation active immunization approach for synucleinopathies: Implications for parkinson's disease clinical trials. Acta Neuropathologica 127 (6):861-879. https://doi.org/10.1007/s00401-014-1256-4

Maroteaux, L., Campanelli, J. T. and Scheller, R. H. 1988. Synuclein: A neuron-specific protein localized to the nucleus and presynaptic nerve terminal. Journal of Neuroscience 8 (8):2804-2815

Matta, S., Van Kolen, K., da Cunha, R., van den Bogaart, G., Mandemakers, W., Miskiewicz, K., De Bock, P.J., Morais, V. A., Vilain, S., Haddad, D., Delbroek, L., Swerts, J., Chavez-Gutierrez, L., Esposito, G., Daneels, G., Karran, E., Holt, M., Gevaert, K., Moechars, D.W., De Strooper, B. and Verstreken, P. 2012. Lrrk2 controls an endoa phosphorylation cycle in synaptic endocytosis. Neuron 75 (6):1008-1021. https://doi.org/10.1016/j.neuron.2012.08.022

Milosevic, I., Giovedi, S., Lou, X., Raimondi, A., Collesi, C., Shen, H., Paradise, S., O'Toole, E., Ferguson, S., Cremona, O. and De Camilli, P. 2011. Recruitment of endophilin to clathrin-coated pit necks is required for efficient vesicle uncoating after fission. Neuron 72 (4):587-601. https://doi.org/10.1016/j.neuron.2011.08.029

Moors, T. E., Hoozemans, J. J., Ingrassia, A., Beccari, T., Parnetti, L., Chartier-Harlin, M. C. and van de Berg, W. D. 2017. Therapeutic potential of autophagy-enhancing agents in parkinson's disease. Molecular Neurodegeneration 12 (1):11. https://doi.org/10.1186/s13024-017-0154-3

Murdoch, J. D., Rostosky, C. M., Gowrisankaran, S., Arora, A. S., Soukup, S. F., Vidal, R., Capece, V., Freytag, S., Fischer, A., Verstreken, P., Bonn, S., Raimundo, N. and Milosevic, I. 2016. Endophilin-a deficiency induces the foxo3afbxo32 network in the brain and causes dysregulation of autophagy and the ubiquitin-proteasome system. Cell Reports 17 (4):1071-1086. https://doi.org/10.1016/j.celrep.2016.09.058

Olanow, C.W. and Brundin, P. 2013. Parkinson's disease and alpha synuclein: Is Parkinson's disease a prion-like disorder? Movement Disorders 28 (1):31-40. https://doi.org/10.1002/mds.25373

Papandreou, M. E. and Tavernarakis, N. 2017. Autophagy and the endo/exosomal pathways in health and disease. Biotechnology Journal 12 (1). https://doi.org/10.1002/ biot.201600175

Parkinson's Disease, 2018. Retreived on April 18, 2018 from https://en.wikipedia.org/wiki/Parkinson\%27s_disease

Pechstein, A., Gerth, F., Milosevic, I., Japel, M., EichhornGrunig, M., Vorontsova, O., Bacetic, J., Maritzen, T., Shupliakov, O., Freund, C. and Haucke, V. 2015. Vesicle uncoating regulated by sh3-sh3 domain-mediated complex formation between endophilin and inter- 
sectin at synapses. EMBO Reports 16 (2):232-239. https://doi.org/10.15252/embr.201439260

Razdorskaya, V. V., Voskresenskaya, O. N., Yudina, G. K. 2016. Parkinson's disease in Russia: prevalence and incidence. Saratov Journal of Medical Scientific Research 12(3):379-384.

Rivero-Rios, P., Madero-Perez, J., Fernandez, B. and Hilfiker, S. 2016. Targeting the autophagy/lysosomal degradation pathway in parkinson's disease. Current Neuropharmacology 14 (3):238-249

Saheki, Y. and De Camilli, P. 2012. Synaptic vesicle endocytosis. Cold Spring Harbor Perspectives in Biology 4 (9):a005645. https://doi.org/10.1101/cshperspect.a005645

Schulz-Schaeffer, W.J. 2012. Neurodegeneration in parkinson disease: Moving lewy bodies out of focus. Neurology 79 (24):2298-2299. https://doi.org/10.1212/ WNL.0b013e318278b6a7

Scott, D. and Roy, S. 2012. Alpha-synuclein inhibits intersynaptic vesicle mobility and maintains recycling-pool homeostasis. Journal of Neuroscience 32 (30):10129-10135. https://doi.org/10.1523/JNEUROSCI.0535-12.2012

Scott, D. A., Tabarean, I., Tang, Y., Cartier, A., Masliah, E. and Roy, S. 2010. A pathologic cascade leading to synaptic dysfunction in alpha-synuclein-induced neurodegeneration. J ournal ofNeuroscience 30 (24):8083-8095. https://doi.org/10.1523/JNEUROSCI.1091-10.2010

Shehadeh, L., Mitsi, G., Adi, N., Bishopric, N. and Papapetropoulos, S. 2009. Expression of lewy body protein septin 4 in postmortem brain of parkinson's disease and control subjects. Movement Disorders 24 (2):204-210. https://doi.org/10.1002/mds.22306

Soukup, S. F., Kuenen, S., Vanhauwaert, R., Manetsberger, J., Hernandez-Diaz, S., Swerts, J., Schoovaerts, N., Vilain, S., Gounko, N. V., Vints, K., Geens, A., De Strooper, B. and Verstreken, P. 2016. A Irrk2-dependent en- dophilina phosphoswitch is critical for macroautophagy at presynaptic terminals. Neuron 92 (4):829-844. https://doi.org/10.1016/j.neuron.2016.09.037

Soukup, S. F. and Verstreken, P. 2017. Endoa/endophilin-a creates docking stations for autophagic proteins at synapses. Autophagy 13 (5):971-972. https://doi.org/10.108 $0 / 15548627.2017 .1286440$

Staras, K., Branco, T., Burden, J. J., Pozo, K., Darcy, K., Marra, V., Ratnayaka, A. and Goda, Y. 2010. A vesicle superpool spans multiple presynaptic terminals in hippocampal neurons. Neuron 66 (1):37-44. https://doi.org/10.1016/j. neuron.2010.03.020

Vargas, K. J., Makani, S., Davis, T., Westphal, C. H., Castillo, P. E. and Chandra, S. S. 2014. Synucleins regulate the kinetics of synaptic vesicle endocytosis. Journal of Neuroscience 34 (28):9364-9376. https://doi.org/10.1523/JNEURO$\mathrm{SCl} .4787-13.2014$

Wang, L., Das, U., Scott, D. A., Tang, Y., McLean, P. J. and Roy, S. 2014. Alpha-synuclein multimers cluster synaptic vesicles and attenuate recycling. Current Biology 24 (19):2319-2326. https://doi.org/10.1016/j.cub.2014.08.027

Woods, W.S., Boettcher, J.M., Zhou, D. H., Kloepper, K.D., Hartman, K. L., Ladror, D.T., Qi, Z., Rienstra, C. M. and George, J.M. 2007. Conformation-specific binding of alpha-synuclein to novel protein partners detected by phage display and nmr spectroscopy. Journal of Biological Chemistry 282 (47):34555-34567. https://doi.org/10.1074/jbc.M705283200

Zhang, Y., Gao, J., Chung, K. K., Huang, H., Dawson, V. L. and Dawson, T. M. 2000. Parkin functions as an e2-dependent ubiquitin- protein ligase and promotes the degradation of the synaptic vesicle-associated protein, cdcrel-1. Proceedings of the National Academy of Science, USA 97 (24):13354-13359. https://doi.org/10.1073/ pnas. 240347797 